\title{
Multiscale Biofluidic and Nanobiotechnology Approaches for Treating Sepsis in Extracorporeal Circuits
}

\author{
Joo H. Kang (iD * \\ Received: 12 February, 2020 / Accepted: 1 March, 2020 / Published online: 12 March, 2020 \\ (C) The Korean BioChip Society and Springer 2020
}

\begin{abstract}
Infectious diseases and their pandemics periodically attract public interests due to difficulty in treating the patients and the consequent high mortality. Sepsis caused by an imbalanced systemic inflammatory response to infection often leads to organ failure and death. The current therapeutic intervention mainly includes "the sepsis bundles," antibiotics (antibacterial, antiviral, and antifungal), intravenous fluids for resuscitation, and surgery, which have significantly improved the clinical outcomes in past decades; however, the patients with fulminant sepsis are still in desperate need of alternative therapeutic approaches. One of the potential supportive therapies, extracorporeal blood treatment, has emerged and been developed for improving the current therapeutic efficacy. Here, I overview how the treatment of infectious diseases has been assisted with the extracorporeal adjuvant therapy and the potential utility of various nanobiotechnology and microfluidic approaches for developing new auxiliary therapeutic methods.
\end{abstract}

Keywords: Microfluidics, Nanobiotechnology, Extracorporeal devices, Infectious diseases, Blood treatment, Sepsis

\section{Introduction}

Sepsis, a systemic inflammatory disease, is caused by an imbalanced overwhelming systemic inflammatory

Department of Biomedical Engineering, Ulsan National Institute of Science and Technology (UNIST), 50, UNIST-gil, Ulsan 44919, Republic of Korea

*Correspondence and requests for materials should be addressed to J.H. Kang (®jookang@unist.ac.kr) response to infection, which often leads to organ failure and death. Because it progresses rapidly while the determination of a cause of infection takes hours to days, the current state of the art treatment of sepsis in hospitals requires careful assessment of patients' conditions, followed by proper treatment based on the "sepsis bundle"1. It was not until the 20th century that Hugo Schottmüller ${ }^{2}$ and Roger Bone $e^{3}$ proposed the modern clinical definition of sepsis. Before this era, sepsis was regarded as severe biological decay that may accompany fevers ${ }^{4}$ or a syndrome that might have resulted from invisible living creatures ${ }^{5,6}$.

This new foundation for sepsis has shifted the direction of the sepsis research from a source of infection to the host inflammatory response, which has led considerable efforts in drug development for anti- inflammatory therapies. Xigris, for example, a recombinant form of human activated protein $\mathrm{C}$ that has anticoagulative and cytoprotective effects was developed $^{7}$. After extensive clinical research that reported no efficacy of Xigris for treating septic patients ${ }^{8}$, Eli Lilly and Co. withdrew Xigris from the market ${ }^{9}$. Despite the lack of proper drugs that effectively mitigate sepsis, the mortality of sepsis has gradually reduced thanks to consolidated efforts of clinicians, such as the Surviving Sepsis Campaign Guidelines, which was recently updated in $2018^{1}$. However, we are still in desperate need of developing a new strategy to fight against infectious outbreaks worldwide whenever infectious diseases, such as severe acute respiratory syndrome (SARS) ${ }^{10}$, Ebola ${ }^{11}$, middle east respiratory syndrome (MERS) ${ }^{12}$, surfaced and threatened the world. In the past five years, we experienced multiple severe outbreaks of Ebola (2014-2016 in West Africa), MERS (2015 in the Republic of Korea), 
and the recent coronavirus outbreak (COVID-19 in Wuhan, China). However, we mainly rely on taking preventative measures against epidemics due to the lack of proper therapeutic strategy in handling severe septic conditions.

Because sepsis is a complicated and dynamic syndrome that involves enormous numbers of parameters that regulate the inflammatory network, it was challenging to inhibit a particular pathway because the disease can still progress by detouring the inhibited pathways as we have already witnessed the case of Xigris. Thus, some of the researchers and clinicians have paid attention to removing a source of infection that persistently triggered a catastrophic inflammatory response for developing an adjuvant therapy. This idea was evidenced by several clinical studies supporting that viral and bacterial loads in the blood are a major contributor to the severity and mortality of sepsis ${ }^{13,14}$. Due to the rapid progress of sepsis and a high frequency $(\sim 70 \%)$ of negative results of blood culture in septic human blood ${ }^{15}$, identification of a source of infection in time is often elusive even in state-of-the-art clinical settings. For this, an auxiliary therapeutic approach could be a preferable treatment option for clinicians while the conventional "sepsisbundle" procedures are mitigating symptoms of severe septic patients. This has led to developing several medical devices that have been available in the market for the past decades. This review will mainly scrutinize extracorporeal blood treatment approaches for treating sepsis and how the researchers in the interdisciplinary fields of nanobiotechnology and biochips could make potential contributions to improving the efficacy of the biomedical devices addressing the unmet needs in sepsis treatment.

\section{Pathogen-capturing Reagents Immobilized on Fiber Surface}

Efforts first dedicated to removing infectious reagents in whole blood of septic patients were developed by modifying the conventional extracorporeal blood treatment devices, such as hemofiltration devices. The inner surface of the fiber bundles of the device was functionalized with synthetic proteins ${ }^{16}$ or antibiotics ${ }^{17}$ that were known to capture a range of Gram-negative pathogens or lipopolysaccharides (LPS) (Figure 1A). The fibers of Toraymyxin ${ }^{\circledR}$ and oXiris ${ }^{\circledR}$, for example, are functionalized with polymyxin B (antibiotics) and polyethyleneimine (PEI) (positively charged mo- lecule), respectively, which are known to bind Gramnegative bacteria and LPS (negatively charged moiety). This approach has been extensively attempted in clinical trials as well as in large animal models because it can be simply integrated with the existing blood filtration devices that had already been approved for treating patients with organ failures. However, the devices based on this principle were more effective in depleting free LPS or pathogen debris probably caused by antibiotic treatment ${ }^{18}$ than removing intact bacteria that were planktonic in whole blood. This is most likely due to the low binding efficiency of relatively large bacterial cells $(\sim 1-2 \mu \mathrm{m}$ in diameter) in comparison to submicrometer-sized LPS molecules. If we consider only stochastic movements of pathogenic materials and capturing matrix, the binding efficiency of planktonic particles in a solution to the capturing matrix is determined by the thermal fluctuation $\left(k_{\mathrm{d}}\right)$ and collisions caused by shear $\left(k_{\text {shear }}\right)^{19}$. Because the collision rate constant of diffusion, $k_{\mathrm{d}}$, is given by

$$
k_{d}=\frac{2 r_{c} k_{b} T}{3 \eta r_{b}}
$$

where $r_{\mathrm{c}}$ is the radius of a pathogen $(\sim 0.5 \mu \mathrm{m})$, and $r_{\mathrm{b}}$ is the radius of a capturing particle, $k_{\mathrm{b}}$ is the Boltzmann constant, $T$ is the temperature $(\mathrm{K})$, and $\eta$ is the fluid viscosity $\left(\mathrm{N} \mathrm{s} \mathrm{m}^{-2}\right)$, the binding efficiency of free bacteria to a solid wall surface via thermal fluctuation where $r_{\mathrm{b}}$ approaches an infinite radius becomes negligible. Thus, physical contact of bacterial cells to the surface is an essential prerequisite to achieve the complete removal of pathogens from blood in this principle.

Moreover, the efficiency of capturing LPS in blood should be significantly higher than whole bacteria because the frequency of LPS encountering the surface is greater than individual intact bacterial cells due to their enormous numbers after being scattered by bactericidal antibiotics. A report published by researchers at Harvard University also demonstrated that the use of antibiotics significantly increases the depletion efficiency of pathogen-associated molecular patterns (PAMPs) in a septic rodent animal mod$\mathrm{el}^{18}$. Thus, it is recommended to use the LPS adsorbing devices, particularly along with antibiotic treatment. However, in the case of infection caused by multidrug-resistant (MDR) or pan-drug resistant (PDR) bacteria where the use of antibiotics is often ineffective, this approach may not be a preferable strategy. 
A
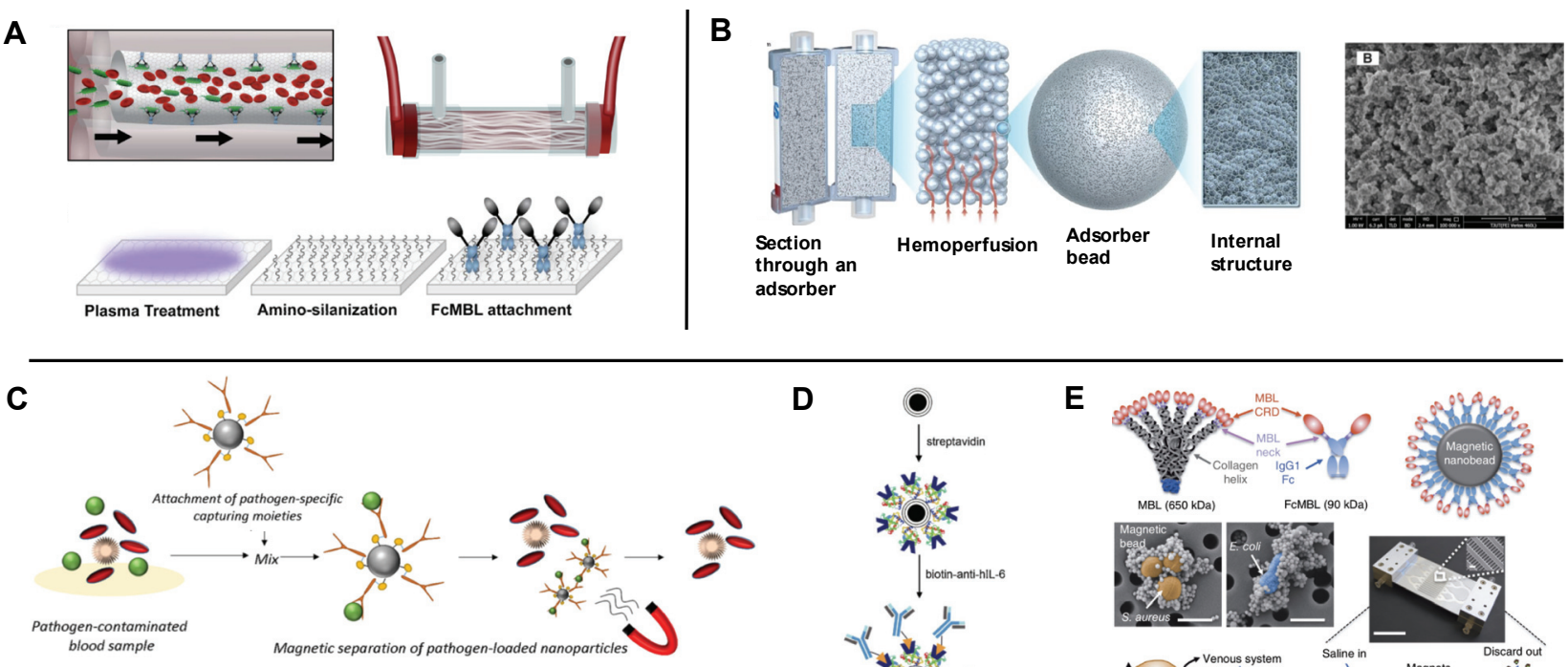

D

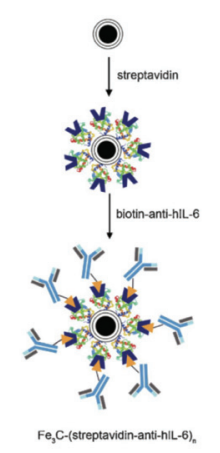

E

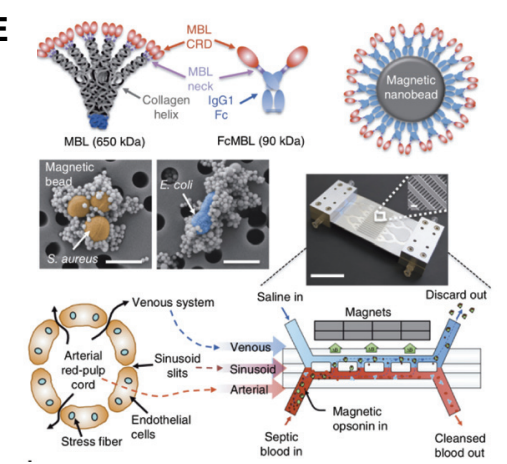

Figure 1. (A) A schematic drawing of surface adsorption-based extracorporeal devices. The inner surface of fibers bundled in the device is functionalized with proteins or synthetic polymers, which are known to capture a wide range of pathogens and toxins in blood $^{18}$. (B) An illustration showing a cross-sectional view of porous beads and their internal structures of the CytoSorb ${ }^{\circledR}$ filter, which was developed for lowering cytokine levels in blood of septic patients ${ }^{20}$. (C, D, E) Magnetic particle-assisted blood treatment approaches. (C) Magnetic particles are functionalized with pathogen-capturing moieties ${ }^{21}$. (D) Magnetic particles coated with anti-IL6 was developed for removing a proinflammatory cytokine in blood ${ }^{22}$. (E) An engineered human FcMBL molecule conjugated on magnetic nanoparticles where the FcMBL molecules were designed to be oriented with carbohydrate recognition domains facing outward $^{23}$.

\section{Porous Polymer Beads Absorbing Cytokines}

The severity of sepsis is often driven by overwhelming amounts of cytokines produced throughout the body, the cytokine storm. Cytokines are a broad category of small proteins responsible for cell to cell signaling $(<40 \mathrm{kDa})$, and their productions and surges in the host immune system are asynchronous. Because a variety of cytokines are responsible for the rapid progress of sepsis, an idea of reducing levels of all small molecules in septic blood was set out to develop for treating septic patients. Hemofiltration (HF) was initially applied to lowering the concentration of cytokines; however, due to the relatively inefficient rate in depleting cytokines, the clinical trial did not find a significantly improved outcome for treating septic patients ${ }^{24}$. After a septic animal study in rats showed that physical adsorption of cytokines (IL-6) to HF filter surfaces was attributed to reducing cytokine levels in blood ${ }^{25}$, the many researchers started to investigate how to maximize physical adsorption of cytokines in an extracorporeal circuit while demon- strating their prototype devices in a small animal mode $^{26}$ and developing mathematical models predicting the cytokine removal efficiency ${ }^{27}$. The most well-known product based on this principle is CytoSorb ${ }^{\circledR}$ in which polymer beads with large amounts of the porous cavity in a range of 250 Angstroms to about 2000 Angstroms ${ }^{28}$ hold up small molecules, such as cytokines, while whole septic blood flows through the device (Figure 1B). However, because pro-inflammatory (IL-1, IL-12, IL-18, TNF- $\alpha$, IFN- $\gamma$ ) and anti-inflammatory (IL-4, IL-6, IL-10, IL-10, IL-13) cytokines finely orchestrate complicated molecular cascades of responses to infection, therapeutic strategies aiming to reduce cytokine levels in blood are still disputable. One of the challenges in this approach is to find the right time to treat patients with adsorbing filters because levels of various cytokines in patients are dynamic and often unpredictable ${ }^{29}$.

\section{Magnetic Nanoparticle-assisted Blood Cleansing Devices}

Magnetic separation is a classical isolation method 
for harvesting a particular fraction of cells, molecules, or impurity from heterogeneous mixtures, such as whole blood ${ }^{30}$, various biological liquid samples ${ }^{31-33}$, and nanotube solutions ${ }^{34}$. This principle has been applied to reducing pathogen levels in whole blood circulating in an extracorporeal circuit in vitro and in vivo animal models (Figure 1C-E). Advantages of the magnetic particles-based blood treatment methods over other competitors described above include that it offers the considerably higher collision rate constant of diffusion $\left(k_{\mathrm{d}}\right.$, Equation (1)) and the larger active surface areas $\left(A_{\text {cap }}\right)$ capturing pathogens $\left(k_{\mathrm{d}}=10^{-17} \sim\right.$ $\left.10^{-18} \mathrm{~m}^{3} / \mathrm{s}, A_{\text {cap }}=\sim 10 \sim 10^{2} \mathrm{~m}^{2} / \mathrm{g}\right)^{19,35}$ in comparison to the surface-adhesion-based bacterial capture method $\left(k_{\mathrm{d}} \rightarrow 0, A_{\text {cap }}=4 \sim 8 \mathrm{~m}^{2}\right)^{36}$. It can also continuously feed fresh capturing reagents into the bloodstream at every moment instead of repassing blood through the capturing surface matrix multiple times, which may gradually compromise the capturing ability. There are several parameters in magnetic blood treatment that determine the pathogen depletion efficiency in septic blood; 1) opsonin molecules that capture a broad range of pathogens without having to carry out prior identification of pathogens, 2) sizes and concentrations of magnetic particles, 3) magnetic forces exerted on the magnetic particle-bacteria complexes, and 4) a design and dimensions of microfluidic channels. Because there is a high degree of variety of carbohydrate chains or proteins expressed on bacteria, viruses, and fungi, it has been challenging to develop a generic opsonin molecule that targets a wide range of pathogens. Researchers initially employed antibody molecules or metal chelators conjugated on magnetic particles to deplete a particular microorganism 37,38 , metal ions $\left(\mathrm{Pb}^{2+}\right)$, drugs, and cytokines (IL-6) ${ }^{22}$ in blood (Figure 1E). These approaches would be practical when we could have beforehand identified a source of infection or intoxication; however, in most infection cases, instantaneous identification of causative pathogens is a daunting task. To address this, Lee, J.-J. et al. developed synthetic ligand zinc-coordinated bis(dipicolylamine) (bis-Zn-DPA)-coated magnetic nanoparticles, which was supposed to form bonds with anionic phospholipids present on the outer surface of both Gram-positive and Gram-negative bacteria (Figure 2A). They also demonstrated its utility using Gram-negative bacteria, E. coli, bound with bis-ZnDPA-coated magnetic nanoparticles ${ }^{39}$. The engineered human opsonin molecule (Fc-mannose binding lectin, FcMBL) was also demonstrated to deplete a broad range of pathogens in whole blood (Figure $2 \mathrm{~A})^{23}$. The second technical issue is how to optimize the size and concentrations of magnetic nanoparticles to maximize the efficiency of the blood cleansing system. Kang, J.H. et al. developed a theoretical model combining an orthokinetic collision model and a magnetophoretic model to predict the optimal size and concentrations of the magnetic nanoparticles that yield the most efficient depletion of pathogens in a buffer solution, plasma, and whole blood, respectively (Figure 2B). ${ }^{19}$ Besides, the magnetic force exerted on the magnetic particles and their complexes bound with pathogens is a determinant of magnetic separation, which is proportional to magnetic flux density gradients along the direction of magnetic pulling. Although an electromagnetic system to pull out magnetic particles offers simplicity for switching the magnetic forces on and off, it appears unfavorable for this particular application because of the limited magnetic flux density gradients due to electromagnetic heating ${ }^{40}$. The permanent magnets assembled with alternating polarization generate the highly enhanced magnetic flux density gradients at a gap adjacent to two assembled magnets ${ }^{23}$. Recently, the Halbach magnetic array was experimentally demonstrated to manipulate magnetic nanoparticles at distances up to 18 $\mathrm{mm}$ and wash magnetic particles used in a multistep-immunoassay process in a deep well plate ${ }^{41}(\mathrm{Fi}-$ gure $2 \mathrm{C}, \mathrm{i}$ ). The ferromagnetic microstructures also have been employed to augment the magnetic flux density gradients to manipulate superparamagnetic nanoparticles that exhibit relatively weak magnetic moments (Figure 2C, ii-iii) ${ }^{34,42}$. Finally, the demonstration of a new design principle for microfluidic channels to remove magnetic particles more effectively in viscous fluids was successfully implemented where secondary spiral flows combined with magnetophoresis significantly enhanced the magnetic separation efficiency even in undiluted whole blood in a continuous manner (Figure 2D) ${ }^{43}$. Given the recent progress and persistent efforts dedicated to magnetically removing pathogens from whole blood, the magnetic separation principle could be one day incorporated in a biomedical device from which many septic patients in dire need benefit in the near future.

\section{Commercial Products Available in the Market and Tested in Clinical Trials}

The extracorporeal biomedical devices that have acquired the approval worldwide for treating septic patients predominantly base on the surface-adsorption principles. (Table 1) Due to their variable and inconclusive therapeutic efficacy, the cost covered by insurance supported either by government or private 


\section{A A generic binding opsonin}

i)

ii)
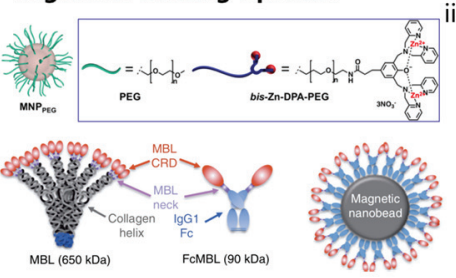

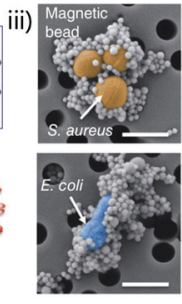

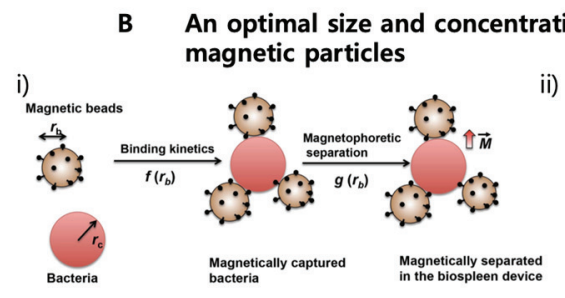

ii)

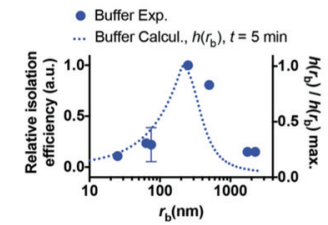

C Magnetic forces
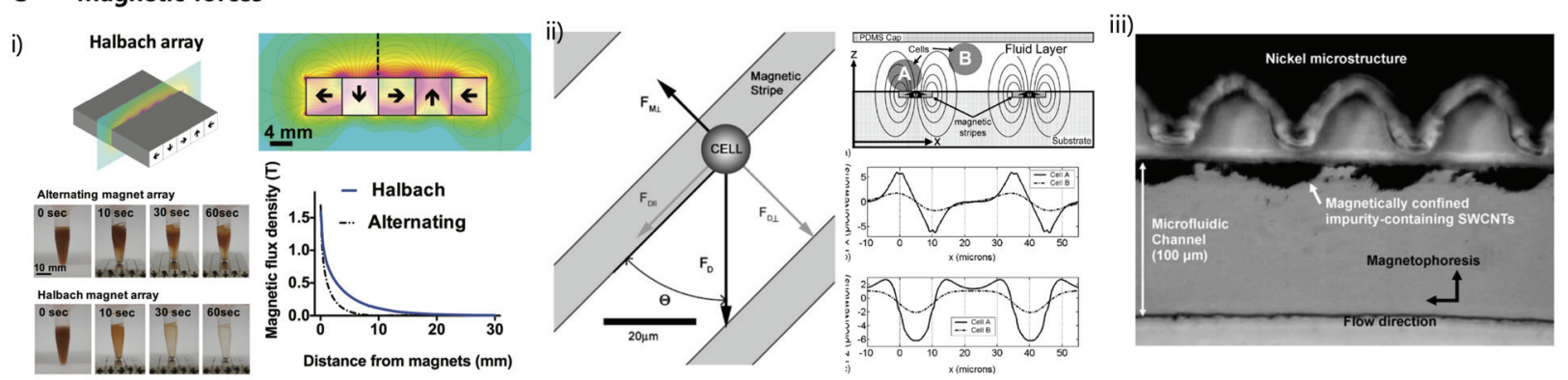

D A design of microfluidic channels

i)

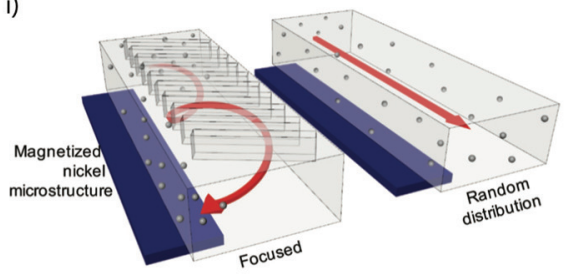

ii)



iii)

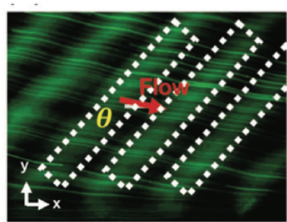

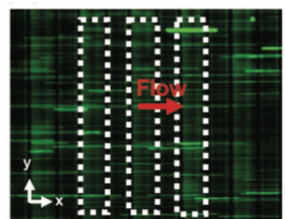

Figure 2. Experimental parameters that determine the magnetic depletion efficiency of pathogens in blood. (A) An opsonin molecule that captures a wide range of pathogens, including Gram-positive, Gram-negative, fungi, and viruses. i) zinc-coordinated bis(dipicolylamine) (bis-Zn-DPA) coated on magnetic particles forms coordination bonds with anionic phospholipids present on pathogen surfaces ${ }^{39}$. ii) An engineered human C-type lectin, mannose-binding lectin (MBL), fused to human IgG1 Fc (FcMBL) ${ }^{23}$. iii) Pseudo-colored SEM images of $S$. aureus (upper) and E. coli (bottom) captured by magnetic nanoparticles coated with Fc-MBL ${ }^{23}$. (B) i) An optimal size and concentration of magnetic particles was predicted by a theoretical model combining an orthokinetic collision and a magnetophoretic model ${ }^{19}$. ii) The theoretical prediction (dotted line) was corroborated by the experimental results (dark circles) ${ }^{19}$. (C) The magnetic forces acting on magnetic particles can be improved by assembling permanent magnets with polarization angles varying clockwise by $90^{\circ}$ (Halbach arrays) ${ }^{41}$ or ii) integrating ferromagnetic microstructures to enhance the magnetic flux density gradients ${ }^{42}$. iii) The nickel microstructures allows carbon nanotubes containing superparamagnetic impurity to be selectively separated in the microfluidic channel when exposed to external magnetic fields ${ }^{34}$. (D) i) Slanted obstacle arrays patterned in a microfluidic channels induce asymmetric pressure gradients across the channel ${ }^{44,45}$, which results in secondary lateral flows dragging the magnetic particles in spiral directions ${ }^{43}$. ii) COMSOL Multiphysics ${ }^{\circledR}$ simulation predicted the deflected paths of the magnetic particles, which correspond to the experimental results (iii, left panel) ${ }^{43}$.

sectors has regional differences; for instance, in the Republic of Korea, use of the Toraymyxin ${ }^{\mathrm{TM}}$ filters is not currently subsidized by general health insurance carriers, while in Japan, it can be reimbursed by the Japanese national health insurance since $1994^{17}$. However, despite insufficient promising results of adjunctive extracorporeal treatment obtained by randomized controlled trials (RCTs), sporadic clinical studies showing positive efficacy of treating septic patients in several cases of the clinical studies ${ }^{46,47}$ keep the devices being used in an intensive care unit. One of the difficulties in pursuing RCTs of extracor- poreal devices treating sepsis is the lack of patients who can be involved in the clinical trials, but this could be resolved when the therapeutic efficacy becomes notably outstanding without controversy. Besides the products shown in Table 1, several startup companies $^{48,49}$ have been striving to bring their technologies to the bedside. (Table 1)

\section{Technical and Experimental Challenges}

The most crucial role of the extracorporeal adjuvant 
Table 1. A summary of extracorporeal devices for treating sepsis.

\begin{tabular}{|c|c|c|c|c|}
\hline Product Name & Principle & Company & Country & Targets of removal \\
\hline oXiris ${ }^{\circledR}$ & \multirow{6}{*}{ Adsorption } & Baxter Int. Inc. & USA & $\begin{array}{l}\text { Endotoxins, cytokines, uremic } \\
\text { toxin }\end{array}$ \\
\hline Toraymyxin ${ }^{T M}$ & & Toray Industries & Japan & $\begin{array}{l}\text { Gram-negative bacteria, } \\
\text { endotoxin }\end{array}$ \\
\hline Alteco ${ }^{\circledR}$ LPS Adsorber & & Alteco Medical AB & Sweden & Endotoxin \\
\hline Seraph $®$ & & ExThera Medical & USA & $\begin{array}{l}\text { Bacteria (MRSA, E.coli, ESBL-K. } \\
\text { pneumoniae, VRE, etc.) }\end{array}$ \\
\hline Cytosorb ${ }^{\circledR}$ & & CytoSorbents & USA & Cytokines \\
\hline Under development & & BOA Biomedical Inc.* & USA & A broad range of pathogens \\
\hline Under development & $\begin{array}{l}\text { Magnetic } \\
\text { particle } \\
\text { separation }\end{array}$ & hemotune* & Switzerland & $\begin{array}{l}\text { Endotoxin, cytokines, drugs, } \\
\text { heavy metals }\end{array}$ \\
\hline
\end{tabular}

* Startup company

therapy is to lower PAMPs levels in whole blood. However, despite the use of the endotoxin activity assay $\left(\mathrm{EAA}^{\mathrm{TM}}\right.$ ) since $2004^{50}$, there is no such a tool approved worldwide for measuring PAMPs levels in human whole blood yet at this moment. Moreover, recent studies reporting the sequencing results of pathogens in septic patient blood reveal that genome sequencing of pathogens in septic blood retains a considerable frequency of false outcomes, particularly when their concentrations are $10 w^{51}$. Because depletion of PAMPs in blood in extracorporeal therapy varies with the effectiveness of antibiotics ${ }^{18}$, the development of rapid diagnostic tools for antibiotic susceptibility test is also required ${ }^{52}$. Thus, the diagnostic techniques and their capability should be preeminently revolutionized for clinicians to achieve successful sepsis therapy. As for the magnetic particlesbased blood-cleansing approach, the biocompatibility of the particles is the priority to address the safety issue in a phase I clinical trial. As the size of magnetic particles approaches to a nanoscale regime, the magnetic removal efficiency of nanoparticles turns out problematic, and their cytotoxicity becomes even more significant. To this end, Hermann et al. demonstrated that carbon-coated magnetic particles might not induce toxicity in a long-term exposure study when the particles were delivered intravenously ${ }^{53}$. Similar approaches where magnetic particles are decorated with various functional materials are encouraged to pursue to address the unmet needs in this particular application in the future. Rapid assessment of residual magnetic nanoparticles remaining in whole blood after blood-cleansing treatment is also critical to ensure that a minimal amount of the nanoparticles return to the patients. However, the currently available tools to quantitate the magnetic nanoparticles or measure the magnetic susceptibility of whole blood require dedicated equipment, such as a superconducting quantum interference device (SQUID) or a vibrating sample magnetometer (VSM), which are often unavailable in a standard research laboratory. Jang, B.H. et al. developed a microfluidic device where they can rapidly assess magnetic particle concentrations in a range of $0.01 \mathrm{mg} / \mathrm{mL}$ to $5 \mathrm{mg} / \mathrm{mL}$ by measuring the diamagnetic repelling velocity of polystyrene microspheres. ${ }^{54}$ Also, Bougas, L. et al. reported a method of detecting sub-picomolar magnetic nanoparticles in flowing complex fluids ${ }^{55}$, which would be useful when integrated with the extracorporeal device to monitor low amounts of the magnetic particles returning to the patients.

While most extracorporeal blood treatment methods have targeted lowering endotoxin or cytokine levels in blood of the septic patients, extracorporeal removal of viral particles has rarely been explored ${ }^{56}$. Although the previously reported opsonin molecule, $\mathrm{FcMBL}^{23}$, is known to bind several virus particles, no data clearly demonstrating the depletion of viruses in human blood are available in public yet. Thus, additional efforts for proving the utility of extracorporeal devices decreasing viral particle concentrations in blood are also required to be established.

Most in vivo animal studies have been demonstrated in rodent sepsis models, including cecal ligation and puncture (CLP) and intravenous or intraperitoneal infection. Before bringing the developed technology to clinical trials, it is essential to test the efficacy in a 
large animal model, such as swine ${ }^{57}$, rabbit, or baboon $^{58}$. However, the current animal models provide poor predictability of human clinical outcomes ${ }^{58}$. Although the current sepsis animal models do not represent pathophysiological outcomes of human septic patients, fortunately, we could still utilize the animal models for developing the extracorporeal blood treatment devices. This is because we only assess a degree of pathogen levels depleted in the blood of septic animals, which may not much differ from the depletion efficiency in human blood, rather than studying pathophysiological or pharmacokinetic studies.

\section{Summary and Outlook}

The research resources and efforts that have been made for studying cancer, cardiovascular diseases, and other diseases have always outweighed those dedicated to infectious diseases. Moreover, the development processes of conventional extracorporeal devices did not actively involve interdisciplinary efforts in the past. As we overviewed above, there are many technical challenges that researchers in interdisciplinary fields ranging from medicine and biology to nanotechnology, polymer sciences, and complex fluid dynamics, could work together to revolutionize the current extracorporeal therapeutic strategies. Besides removing pathogenic materials from blood, elimination of activated leukocytes may have positive impacts on therapeutic efficacy ${ }^{59}$; however, more fundamental and clinical evaluations are required to follow because the detailed primary mechanisms and their consequences governing sepsis progression remain largely unknown ${ }^{16}$. It is also important to note that once proving their therapeutic efficiency in RCTs, it would be worth exploring whether the extracorporeal therapeutic scenario could not only help decrease the mortality of septic patients but also improve the post-therapeutic life quality because about half of the survivors of sepsis suffer from the post-sepsis syndrome ${ }^{60}$.

Acknowledgements This work was supported by a grant funded by Samsung Research Funding and Incubation Center for Future Technology (SRFC-IT16 02-02), and the National Research Foundation of Korea (NRF) grant funded by the Korea government (MSIT) (Grant No. NRF-2019R1C1C1006124).

Conflict of Interests The authors declare no competing financial interests.

\section{References}

1. Levy, M.M., Evans, L.E. \& Rhodes, A. The surviving sepsis campaign bundle: 2018 update. Intensive Care Med. 44, 925-928 (2018).

2. Gary, T., Mingle, D. \& Yenamandra, A. The evolving definition of sepsis. Int. Clin. Pathol. J. 2, 154159 (2016).

3. Bone, R.C., Balk, R.A., Cerra, F.B., Dellinger, R.P., Fein, A.M., Knaus, W.A., Schein, R. \& Sibbald, W. J. Definitions for sepsis and organ failure and guidelines for the use of innovative therapies in sepsis. The ACCP/SCCM Consensus Conference Committee. American College of Chest Physicians/Society of Critical Care Medicine. CHEST Journal 101, 1644-1655 (1992).

4. Rittirsch, D., Flierl, M.A. \& Ward, P.A. Harmful molecular mechanisms in sepsis. Nat. Rev. Immunol. 8, 776-787 (2008).

5. Baron, R.M., Baron, M.J. \& Perrella, M.A. Pathobiology of Sepsis: Are We Still Asking the Same Questions? Am. J. Respir. Cell Mol. Biol. 34, 129-134 (2006).

6. Botero, J.S.H \& Perez, M.C.F. The history of sepsis from ancient Egypt to the XIX century. in Sepsis An Ongoing and Significant Challenge (ed. Azevedo, L.) DOI 10.5772/51484 (2012).

7. Abraham, E., Laterre, P.-F., Garg, R., Levy, H., Talwar, D., Trzaskoma, B.L., François, B., Guy, J.S., Brückmann, M., Rea-Neto, Á., Rossaint, R., Perrotin, D., Sablotzki, A., Arkins, N., Utterback, B.G. \& Macias, W.L. Drotrecogin alfa (activated) for adults with severe sepsis and a low risk of death. N. Engl. J. Med. 353, 1332-1341 (2005).

8. Ranieri, V.M., Thompson, B.T., Barie, P.S., Dhainaut, J.F., Douglas, I.S., Finfer, S., Gårdlund, B., Marshall, J.C., Rhodes, A. \& Artigas, A. Drotrecogin alfa (activated) in adults with septic shock. N. Engl. J. Med. 366, 2055-2064 (2012).

9. FDA Drug Safety Communication: Voluntary market withdrawal of Xigris [drotrecogin alfa (activated)] due to failure to show a survival benefit (https:// www.fda.gov/drugs/drug-safety-and-availability/fda -drug-safety-communication-voluntary-market-with drawal-xigris-drotrecogin-alfa-activated-due).

10. Peiris, J.S.M., Yuen, K.Y., Osterhaus, A.D.M.E. \& Stöhr, K. The Severe Acute Respiratory Syndrome. N. Engl. J. Med. 349, 2431-2441 (2003).

11. Ilunga Kalenga, O., Moeti, M., Sparrow, A., Nguyen, V.-K., Lucey, D. \& Ghebreyesus, T.A. The ongoing Ebola epidemic in the Democratic Republic of Congo, 2018-2019. N. Engl. J. Med 381, 373-383 (2019).

12. Arabi, Y.M., Balkhy, H.H., Hayden, F.G., Bouchama, A., Luke, T., Baillie, J.K., Al-Omari, A. Hajeer, A.H., Senga, M., Denison, M.R., Nguyen-Van-Tam, J.S., 
Shindo, N., Bermingham, A., Chappell, J.D., van Kerkhove M.D. \& Fowler, R.A. Middle East respiratory syndrome. N. Engl. J. Med 376, 584-594 (2017).

13. Chuang, Y.-C., Chang, S.-C. \& Wang, W.-K. High and increasing Oxa-51 DNA load predict mortality in Acinetobacter baumannii bacteremia: implication for pathogenesis and evaluation of therapy. PLoS ONE 5, e14133 (2010).

14. Towner, J.S., Rollin, P.E., Bausch, D.G., Sanchez, A., Crary, S.M., Vincent, M., Lee, W.F., Spiropoulou, C.F., Ksiazek, T.G., Lukwiya, M., Kaducu, F., Downing, R. \& Nichol, S.T. Rapid diagnosis of Ebola hemorrhagic fever by reverse transcriptionPCR in an outbreak setting and assessment of patient viral load as a predictor of outcome. J. Virol.78 4330-4341 (2004).

15. Chung, H., Lee, J.H., Jo, Y.H., Hwang, J.E. \& Kim, J. Circulating monocyte counts and its impact on outcomes in patients with severe sepsis including septic shock: SHOCK 51, 423-429 (2019).

16. Monard, C., Rimmelé, T. \& Ronco, C. Extracorporeal blood purification therapies for sepsis. Blood Purif. 47, 1-14 (2019).

17. Shoji, H. Extracorporeal endotoxin removal for the treatment of sepsis: endotoxin adsorption cartridge (Toraymyxin). Ther. Apheresis Dial. 7, 108-114 (2003).

18. Didar, T.F., Cartwright, M.J., Rottman, M., Graveline, A.R., Gamini, N., Watters, A.L., Leslie, D.C., Mammoto, T., Rodas, M.J., Kang, J.H., Waterhouse, A., Seiler, B.T., Lombardo, P., Qendro, E.I., Super, M. \& Ingber, D.E. Improved treatment of systemic blood infections using antibiotics with extracorporeal opsonin hemoadsorption. Biomaterials 67, 382392 (2015).

19. Kang, J.H., Um, E., Diaz, A., Driscoll, H., Rodas, M. J., Domansky, K., Watters, A.L., Super, M., Stone, H.A. \& Ingber, D.E. Optimization of pathogen capture in flowing fluids with magnetic nanoparticles. Small 11, 5657-5666 (2015).

20. Cytosorbents-How it works, https://www.mddionline. com/file/cytosorbents-how-it-worksjpg.

21. Herrmann, I.K., Schlegel, A.A., Graf, R., Stark, W.J. \& Beck-Schimmer, B. Magnetic separation-based blood purification: a promising new approach for the removal of disease-causing compounds? J. Nanobiotechnol 13, 49 (2015).

22. Herrmann, I.K., Urner, M., Koehler, F.M., Hasler, M., Roth-Z'Graggen, B., Grass, R.N., Ziegler, U., Beck-Schimmer, B. \& Stark, W.J. Blood purification using functionalized core/shell nanomagnets. Small 6, 1388-1392 (2010).

23. Kang, J.H., Super, M., Yung, C.W., Cooper, R.M., Domansky, K., Graveline, A.R., Mammoto, T., Berthet, J.B., Tobin, H., Cartwright, M.J., Watters, A. L., Rottman, M., Waterhouse, A., Mammoto, A.,
Gamini, N., Rodas, M.J., Kole, A., Jiang, A., Valentin, T.M., Diaz, A., Takahashi, K. \& Ingber, D. E. An extracorporeal blood-cleansing device for sepsis therapy. Nat. Med. 20, 1211-1216 (2014).

24. Cole, L. Bellomo, R., Hart, G., Journois, D., Davenport, P., Tipping, P. \& Ronco, C. A phase II randomized, controlled trial of continuous hemofiltration in sepsis. Crit. Care Med. 30, 100-106 (2002).

25. Kellum, J.A. \& Dishart, M.K. Effect of hemofiltration filter adsorption on circulating IL-6 levels in septic rats. Crit. Care 6, 429-433 (2002).

26. Song, M., Winchester, J., Albright, R.L., Capponi, V.J., Choquette, M.D. \& Kellum, J.A. Cytokine removal with a novel adsorbent polymer. Blood Purif. 22, 428-434 (2004).

27. DiLeo, M.V., Kellum, J.A. \& Federspiel, W.J. A simple mathematical model of cytokine capture using a hemoadsorption device. Ann. Biomed. Eng. 37, 222-229 (2009).

28. Young, W.-T., Albright, R.L., Golobish, T.D., Capponi, V. \& Chan, P. Size- selective polymer system, US $8211310 \mathrm{~B} 2$.

29. Shukla, A.M. Extracorporeal therapy in sepsis: are we there yet? Kidney Int. 81, 336-338 (2012).

30. Oh, S., Jung, H., Seo, H., Min, M.-K., Kim, B., Hahn, Y.K., Kang J.H. \& Choi, S. Magnetic activated cell sorting (MACS) pipette tip for immunomagnetic bacteria separation. Sens. Actuators, B 272, 324-330 (2018).

31. Wilson, S., Lane, A., Rosedale, R. \& Stanley, C. Concentration of Mycobacterium tuberculosis from sputum using ligand-coated magnetic beads. Int. $J$. Tuberc. Lung. Dis. 14, 1164-1168 (2010).

32. Kang, J.H., Krause, S., Tobin, H., Mammoto, A., Kanapathipillai, M. \& Ingber, D.E.. A combined micromagnetic-microfluidic device for rapid capture and culture of rare circulating tumor cells. Lab Chip 12, 2175-2181 (2012).

33. Song, S.-H., Lee, J.H., Yoon, J. \& Park, W. Functional microparticle R\&D for IVD and cell therapeutic technology: Large-scale commercialized products. BioChip J. 13, 95-104 (2019).

34. Kang, J.H. \& Park, J.-K. Magnetophoretic continuous purification of single-walled carbon nanotubes from catalytic impurities in a microfluidic device. Small 3, 1784-1791 (2007).

35. Sari, A.Y., Eko, A.S., Candra, K., Hasibuan, D.P., Ginting, M., Sebayang, P. \& Simamora, P. Synthesis, properties and application of glucose coated $\mathrm{Fe}_{3} \mathrm{O}_{4}$ nanoparticles prepared by co-precipitation method. IOP Conf. Ser.: Mater. Sci. Eng. 214, 012021 (2017).

36. Scott, K. Introduction to membrane separations. in Handbook of Industrial Membranes (ed. Scott, K.) 3-185 (Elsevier Science) DOI 10.1016/B978-18561 7233-2/50004-0 (1995). 
37. Xia, N., Hunt, T., Mayers, B., Alsberg, E., Whitesides, G., Westervelt, R. \& Ingber, D. Combined microfluidic-micromagnetic separation of living cells in continuous flow. Biomed. Microdevices 8, 299308 (2006).

38. Yung, C.W., Fiering, J., Mueller, A.J. \& Ingber, D.E. Micromagnetic-microfluidic blood cleansing device. Lab Chip 9, 1171-1177 (2009).

39. Lee, J.-J., Jeong, K.J., Hashimoto, M., Kwon, A.H., Rwei, A., Shankarappa, S.A., Tsui, J.H. \& Kohane, D.S. Synthetic ligand-coated magnetic nanoparticles for microfluidic bacterial separation from blood. Nano Lett. DOI 10.1021/nl3047305 (2013).

40. Microfluidic magnetic particle sorting and separation: a short review. https://www.elveflow.com/mic rofluidic-tutorials/microfluidic-reviews-and-tutorials/ magnetic-particle-separation-a-short-review/.

41. Kang, J.H., Driscoll, H., Super, M. \& Ingber, D.E. Application of a Halbach magnetic array for longrange cell and particle separations in biological samples. Appl. Phys. Lett. 108, 213702 (2016).

42. Inglis, D.W., Riehn, R., Austin, R.H. \& Sturm, J.C. Continuous microfluidic immunomagnetic cell separation. Appl. Phys. Lett. 85, 5093-5095 (2004).

43. Jung, S.H., Hahn, Y.K., Oh, S., Kwon, S., Um, E., Choi, S. \& Kang, J.H. Advection flows-enhanced magnetic separation for high-throughput bacteria separation from undiluted whole blood. Small 14, e1801731 (2018).

44. Chung, A.J. A minireview on inertial microfluidics fundamentals: Inertial particle focusing and secondary Ffow. BioChip J. 13, 53-63 (2019).

45. Choi, S. \& Park, J.-K. Continuous hydrophoretic separation and sizing of microparticles using slanted obstacles in a microchannel. Lab Chip 7, 890-897 (2007).

46. Chang, T., Tu, Y.-K., Lee, C.-T., Chao, A., Huang, C.-H., Wang, M.-J. \& Yeh, Y.-C. Effects of polymyxin $\mathrm{B}$ hemoperfusion on mortality in patients with severe sepsis and septic shock: A systemic review, meta-analysis update, and disease severity subgroup meta-analysis. Crit. Care Med. 45, e858-e864 (2017).

47. Klein, D.J., Foster, D. Walker, P.M., Bagshaw, S.M., Mekonnen, H. \& Antonelli, M. Polymyxin B hemoperfusion in endotoxemic septic shock patients without extreme endotoxemia: a post hoc analysis of the EUPHRATES trial. Intensive Care Med. 44, 22052212 (2018).

48. Hemotune. https://www.hemotune.ch/.
49. BOA Biomedical Inc. https://otd.harvard.edu/news/ new-startup-boa-biomedical-launches-to-reduce-sep sis-deaths.

50. Romaschin, A.D., Klein, D.J. \& Marshall, J.C. Bench-to-bedside review: Clinical experience with the endotoxin activity assay. Crit. Care 16, 248 (2012).

51. Salter, S.J., Cox, M.J., Turek, E.M., Calus, S.T., Cookson, W.O., Moffatt, M.F., Turner, P., Parkhill, J., Loman, N.J. \& Walker, A.W. Reagent and laboratory contamination can critically impact sequencebased microbiome analyses. BMC Biol. 12, 87 (2014).

52. Kim, S., Masum, F. \& Jeon, J.S. Recent developments of chip-based phenotypic antibiotic susceptibility testing. BioChip J. 13, 43-52 (2019).

53. Herrmann, I.K., Beck-Schimmer, B., Schumacher, C.M., Gschwind, S., Kaech, A., Ziegler, U., Clavien, P.-A., Günther, D., Stark, W.J., Graf, R. \& Schlegel, A.A. In vivo risk evaluation of carbon-coated iron carbide nanoparticles based on short- and long-term exposure scenarios. Nanomedicine 11, 783-796 (2016).

54. Jang, B.H., Kwon, S. \& Kang, J.H. Measurement of the magnetic susceptibility of subtle paramagnetic solutions using the diamagnetic repulsion of polymer microparticles. Lab Chip 19, 2356-2361 (2019).

55. Bougas, L., Langenegger, L.D., Mora, C.A., Zeltner, M., Stark, W.J., Wickenbrock, A., Blanchard, J.W. $\&$ Budker, D. Nondestructive in-line sub-picomolar detection of magnetic nanoparticles in flowing complex fluids. Sci. Rep. 8, 3491 (2018).

56. Shahidi Bonjar, A.H. Antiviral therapy: a perspective. Drug Des., Dev. Ther. DOI 10.2147/DDDT.S83037 (2016).

57. Park, I., Lee, J.H., Jang, D.-H., Kim, D., Chang, H., Kwon, H., Kim, S., Kim, T.S. \& Jo, Y.H. Characterization of fecal peritonitis-induced sepsis in a porcine model. J. Surg. Res. 244, 492-501 (2019).

58. Fink, M.P. Animal models of sepsis. Virulence 5, 143-153 (2014).

59. Pino, C.J., Yevzlin, A.S., Tumlin, J. \& Humes, H.D. Cell-based strategies for the treatment of kidney dysfunction: a review. Blood Purif. 34, 117-123 (2012).

60. Huang, C.Y., Daniels, R., Lembo, A., Hartog, C., O'Brien, J., Heymann, T., Reinhart, K., Nguyen, H. B. \& Sepsis Survivors Engagement Project (SSEP). Life after sepsis: an international survey of survivors to understand the post-sepsis syndrome. Int. J. Qual. Health Care 31, 191-198 (2019). 